\title{
Sachregister zu Band 87
}

Die îtt gedruckten Zahlen bedeuten Eigenarbeiten. Bb. = Buchbesprechungen.

A

Ablatio retinae, Vererbung der

$-\quad 168$

- $\quad$ nach Episkleritis 228

- Kataraktbildung nach Igni-punktur der - 144

- nach Zyklodialyse 52Acusticus-Neurinom 335Adiesche Krankheit und Pu-

pillotonie 169 Alkoholneuritis 319 Angioidstreifen der Netzhaut,

Veränderungen in der Macula

bei - 60 Angiomatosis des Zentralnerven-

systems und der Netzhaut 234,

35o Arsenvergiftung, Erblindung

durch - 319 Augenbewegungen 213 Augenlidspalte, Richtung der -

bei Syphilis congenita 104 Augenmuskellähmungen 221 Augenmuskeln, Veränderungen

an den Arterien der äußeren -

bei Periarteriitis nodosa 294 Auto- oder Homokeratopla-

stik 15 Avitaminose und Neuritis optica

$3 \mathrm{i} 8$

B Basedowsche Krankheit, Ent-

stehung des Exophthalmus bei

- $\quad$ 151Bewegungsapparat des Auges

213 (Bericht) Bindehaut s. Conjunctiva Blindenstatistik in Böhmen 63

(Bb.) Blutdruck, Verhältnis des - zum

intraokularen Druck 65 Brille, stenopäische - - für Schüt-

zen 166

- $\quad$ Tragrand- - für Staroperierte170

Brillenbestimmung und Ver-

ordnung in U.S.A. 37 Buergersche Krankheit 170

C Carotisfunktion, Prüfung der - durch Blutdruckmessungen bei pulsierendem Exophthalmus 354- 357

Chi as ma 323 (Bericht)

Circulus arteriosus iridis major, Wandveränderungen des - bei verschiedenen Formen von

Uveitis 239

Conjunctiva, Behandlung der knotigen Tuberkulose der -165

Conjunctivitis, allergische, nicht infektiöse 172

Cornea, großes subepitheliales Hä-matom der - 131

- $\quad$ Tuberkulose der - 355Cyklin 58

D

Diathermie bei Glaukom 50 
Divergenz 216

Doryl 49

Druck, intraokularer, Verhältnis des allgemeinen Blutdruckes und des Druckes in den Netz-

hautgefäßen zum - 65

Dysostosis craniofacialis 167

Dyszephalodaktylie und uveale Entwicklungsstörung 229

$\mathrm{E}$

Elliotsche Trepanation 51 Endangitis obliterans Wini-

water 170 Endophlebitis obliterans 163 Enophthalmus beim Horner-

schen Syndrom 152 Enzephalitis, Erkrankungen

nach - 225 Enzephalomyelitis dissemi-

nata, optische Varietät der --

$33 \theta$ Epinephrin-Bitartrat 50 Episkleritis, Netzhautablösung

nach - 228 Ergotamin 50 Exkavation, physiologische -

und Schaltgewebe 1 Exophthalmus, pulsierender 151

- $\quad$ Blutdruckmessungen bei - 354,357

$\mathrm{F}$

Feuerlamelle 161

Feuerstar 142

Filaria loa 162, 347

Fissura orbitalis superior 228

Fixationsnystagmus 309

Sachregister zu Band 87.

361

$\theta$

Gehirntumoren, Augenerschei-

nungen bei - 334 - Polyopie bei okzipitalen - 172 Geschichte der Augenheilkunde

133 Glaskörper 149 (Bericht) - Zerfallsvorgänge im - in Be-

ziehung zu Netzhautrissen 355 Glaskörperabhebung 161 Glaskörperstrang, pendelnder

199 Glaukom 43 (Bericht) - ohne Hochdruck 62 Glaukosan 50 Glioma retinae 165 Glutathion in der Linse 139 Goldchloridtätowierung 163 Gradenigosches Syndrom 225 Gynergen 50

H Hand-Schüller-Christiansche

Krankheit 155 Hemianopsie, binasale 325 Hemicrania vasomotoria, Au-

generscheinungen bei - 341 Heterocliromie, neurogene 57 Heterophorie 215 Höhlenplastik mit entzweigeteil-

tem Hautlappen 114 Homo- oder Autokeratopla-

stik 15 Hornersches Syndrom, Enoph-

thalmus beim - 152 Hornhaut s. Cornea Hydrophthalmus 55 Hypophysistumoren, Optikus-

atrophie bei - 184, 324, 336

I

Idiotie, familiäre amaurotische -

und Niemann-Picksche Lipoi-

dose 170 Innere Sekretion und Glaukom

46 - und Katarakt 141 Iridenkleisis 53 Iris 57 (Bericht) Irisatrophie, akute $348 \bullet-$ Bedeutung

der $\mathbf{\square}-$ für die Ent- 
stehung des Glaukoms 47 Iriskammerzysten, Behand-

lung der - 348 Iritis, metastatische - bei Spon-

dylarthritis ankylopoetica 166

$\mathrm{J}$

Jodschäden 358

$\mathrm{K}$

Katarakt $138 \mathrm{ft}$.

- senile hintere Schalen -77

Kataraktoperation 166, 167

- bei Glaukom 48Keimplasma bei Zwillingen 160Keratitis parenchymatosa, nicht luische 236 Keratitis pustuliformis pro-

funda 89 Keratokonjunktivitis sicca

235

Keratoplastik, Homo- oder Auto-K. - 15

Kolipanophthalmie, metastatische 171

Konvergenz 216

Kristallbildung in der Linse 228

Kurzwellen, Wirkungen der -· am Auge 171

$\mathrm{L}$

Linse 138 (Bericht)

- $\quad$ Kristallbildung in der - 226Linsenkapselhäutchen, Vogt-

sches - bei Glaukom 49 Linsenkern, Resorptionserschei-nungen an einem bei der Star-operation

zurückgebliebenen -

356

Lipoidose, Niemann-Picksche - und familiäre amaurotische Idiotie 170

Lochbildung der Macula 164

Lupus erythematodes corneae 160

Lymphadenose, aleukämische der Orbita 153

$\mathrm{M}$

Macula, Affektion der - · mit ab-normer Kopfbehaarung 167

Veränderungen in der - bei Angioidstreifen in der Netzhaut 60

scheibenförmige Degeneration

der - 352 - Pseudotumor der - 161, 173 Malariabehandlung der tabi-

schen Optikusatrophie 298, 322 Membrana pupillaris persi-

stens 57 Menformonbehandlung der Re-

tinitis pigmentosa 26 Meningiome 337 Mikrophakie 54 Musculus obliquus inferior, Sarkom des - 225 Multiple Sklerose und Neuritis

retrobulbaris 316, 317, 331 Myocampter 162 Myopie, Entstehungsweise der -

229

362

Sachregister zu Band 87.

Myopieoperation nach Fukala 146

N Nebenhöhlenerkrankungen

155,156

- $\quad$ und Neuritis retrobulbaris 315Neophangläser 358Nervenkrankheiten und Auge 
329 (Bericht) Nervus nasociliaris 156 Nervus opticus 315 (Bericht)

feinere Struktur des - 232 - - bei Hypophysistumoren 184

retrobulbäre 347

atrophie, Malariabehandlung

der tabischen - 298, 322

Netzhaut s. Retina

Neuritis retrobulbaris bei Ne-benhöhlenaffektion 156, 315

Neuromyelitis optica 330

Nystagmus 308 (Bericht)

0

Ophthalmia sympathica 232,

234 - meningeale Reizerscheinungen

bei - 357 Ophthalmoplegie 224 Orbita 151 (Bericht) Orbitalphlegmone 152

$\mathrm{P}$

Panophthalmie, metastatische

Koli - 171 Pantocain, Schädigungdes Horn-

hautepithels durch - 174 Periarteriitís nodosa 294 Periphlebitis obliterans 163 Periphlebitis

retinae 349 Polyopie bei Tumoren des Okzi-

pitalhirns 172 Pupillenanomalien, nichtsyphi-

litische 63 Pupillotonie 62, 340 - und Adiesche Krankheit 169

$\mathrm{R}$

Refraktion 225

- kindliche 162, 165

- $\quad$ Anomalien der - und Verer-bung 346

Retina, Angiomatosis der - 234 - faserige Zerschlissenheit der

167 - Vererbung der Degeneratio pig-

mentosa der - 228 Retinablutungen, Bedeutung d.

- für Diagnose und Therapie

des Allgemeinleidens 175 Retinagefäße, Verhältnis des

Druckes in den - zum intra-

okularen Druck 65

Retinarisse 203

Retina reflex, nasaler 156

- $\quad$ in Beziehung zu Zerfallsvorgän-gen im Glaskörper 355

Retinitis albuminurica 352 Retinitis Coats 352 Retinitis pigmentosa, Menfor-monbehandlung der $-26$

S Sarkommetastasen, intraoku-

läre 265 Schielen 2i7ff. Schalenkatarakt, seniler hinte-

rer 77 Schaltgewebe und physiologische

Exkavation 1 Schwangerschaft, Gesichtsfeld-

veränderungen in der - 324 Sehbahn 315 (Bericht) Sehnerv s. Nervus opticus

Sexualhormonbestimmungen

im Harn 26 Sphärophakie 54 Ski era, hintere Ziliarnervenschlei-

fen der - 284 Sklerektomie 53 Spondylarthritis ankylopoe-

tica, metastatische Iritis bei -

166 Status dysraphicus 341 Stauungspapille, Pathogenesed. 
- $\quad$ 322Sympathektomie der Carotis bei

Sehnervenatrophie 322 Sympathicus, Beziehungen des

- $\quad$ zu den Ziliarnerven 232Syphilis und Auge 329Syphilis congenita, Richtung

der Augenlidspalte bei $\mathbf{-}-104$

$\mathrm{T}$

Tabaksamblyopie 318 Tätowierung mit Goldchlorid 163 Thalliumschädigungen des Auges 320 Tragrandbrille für Staroperierte

170 Tränenpünktchen, dreifaches

unteres 209 Trichloräthylen, toxische Wir-

kung des - 358 Tuberkulose, prophylaktische

Maßnahmen gegen - 357 Tuberkulose des Auges 168 - - der Cornea 355

- Behandlung der knotigen - derBindehaut 165

Turmschädel 155 - Sehnervenschädigungen bei -321

Namenregister zu Band 87.

363

epi-

$\mathrm{U}$

Uveitis, Wandveränderungen des Circulus arteriosus iridis major bei verschiedenen Formen von $-239$

W

Glaukom bei

Vitamin C in der Linse 138 Vollsalzschäden 358

"Wassersucht, demischer -

Wetter, Einfluß des - auf die Entstehung von Augenkrank-heiten 162

Z

Ziliarkörper 57 (Bericht) Ziliarnerven, Beziehungen des

Sympathicus zu den - 232 Ziliarnervenschleif en, hintere

- der Sklera 284 Zonulalamelle, senile Verschup-

pung der - 161 Zwillinge, Keimplasma bei --160 Zyklodialyse 52

Namenregister zu Band 87.

A

Abeloos 51 Abramowicz 143 Adam 174 Adson 151, 321 Agnello 58, 152 Aliquò-Mazzei 221

Amin 220 Ammann 160 Amsler 141, 145, 162,

164 Amstislavskij 146 Archangelskij 49 Armstrong 217 Arslan 312, 314 Ashikaga 329 Asian

Perez 153 Atkinson 145 Attiah 224 Aubaret 155 Averbach 53

$\mathrm{B}$

Bahr 215 Bailliart 45 Balado 324, 327, 328 Bard 49 Barr 57 Bartels 310 Barth 155 Baudouin 325

Baumann 151 Baurmann 149, 323,

354. 355. 357 Becker 54 Behmann 347 Behr 155 Bencini 146 Benda 334 Bendenritter 228

Benedict 153 Bertotto 58

Bielschowski 215,

216, 219, 222 Bietti 139, 140, 141,

156 Birch 139

Birch-Hirschfeld43 Biró 228 Bistis 57 
BlagovescenskiJ2i6 Blaickner 145 Blake 145 Blamwell 339 Blaskovics 145 Blatt 144 Blessig 146 Bocage 329 Böck 43, 223 Bodechtel 328 Bogaert 224, 333, 341 Böhm 139, 140 Bonnet 60, 154 Bonorino 225 Boros Béla 317 Borsellino 149 Boschi 224 Bossalino 59 Botteri 59 Bourquin 162, 164 Brain 317 Bramwell 222, 341 Brandenburg 219 Braun 139, 140, 218 Brecher 214, 310 Britti 316 Brody 326 Bröer 140, 311 Bruckner 162, 164, 165, 168 Brugi 326 Bucklers 144, 314 Brunner 312 Bunge 26 Buschke 138 Busi 155

C Caocci 224 Carteni 139 Cattaneo 144 Cavara 155 Cepero 59 Cestan 330 Chaikoff 142 Chavasse 220 Chonnet 58 Claes 152 Clark 326 Claus 152 Cohen 149, 153 Colavizi 320 Colrat 222 Comas 59 Coppez 59 Corcelle 357 Cordero 156 Cornet 152, 153 de Cori 44 Corrado 218 Cosmettatos 57 Courtis 49, 58 Crvaric 55 Csapody, I. 114, 154, 230 v. Csapody-Mócsy

217 Csillag 145, 229 Cunningham 225 Custodis 59

D. D Dalsgaard-Nielsen 150 Dann 139 Davis 220 Debenedetti 155 Debré 218 